\title{
Supervisor de psicologia clínica: um professor idealizado?
}

\author{
Nancy Ramacciotti de Oliveira-Monteiro ${ }^{1}$ - Universidade Federal de São Paulo, Brasil \\ Maria Lucia Tiellet Nunes - Pontifícia Universidade Católica do Rio Grande do Sul, Porto Alegre, Brasil
}

\begin{abstract}
Resumo
A supervisão de estágios em Psicologia Clínica, fundamental para a formação do aluno de Psicologia, carece de mecanismos de avaliação sistemática, relativa ao supervisor. Este artigo apresenta um estudo que explorou percepções de estagiários de Psicologia Clínica acerca de seus supervisores e da supervisão. O levantamento utilizou uma escala de tipo Likert de cinco pontos e 27 itens. Foram pesquisados 56 alunos de $9^{\circ}$ semestre de curso de graduação em Psicologia, que faziam estágios sob supervisão semanal, há um semestre. Sob a ótica dos supervisionandos, as avaliações foram quase que exclusivamente positivas, a despeito da abordagem teórico-técnica do supervisor. Essa positividade foi discutida em relação a: características do processo ensino-aprendizagem clínico, em especial quanto a formas de avaliação utilizadas; possíveis idealizações; e momento de transição alunoprofissional em que o supervisionando se encontra. É apontada a necessidade de discussões sobre avaliação de professores-supervisores em Psicologia-Clínica.

Palavras-chave: Supervisão; Psicologia clínica; Escala de avaliação do supervisor.
\end{abstract}

\section{Clinical psychology advisor: an idealized master?}

\begin{abstract}
Supervision for undergraduate trainees in clinical psychology - even though fundamental for their training - lacks systematic evaluation mechanisms in regard to the role and function of the supervisor. This paper presents a study taking aim at exploring clinical psychology trainees' perceptions about their supervisors and supervision. The survey was carried out using a five points Likert scale comprising 27 items. The sample was formed by 56 undergraduate students belonging to the ninth semester of Psychology School, undergoing their training under weekly supervision for at least one semester. From the point of view of the trainees under supervision, the evaluations were almost exclusively positive, in spite of theoretical orientation of the supervisor. This positive result is discussed in relation to: characteristics of the clinical learning process - in special regarding the evaluation forms used; possible idealization; and the moment of transition from student to professional, in which the undergraduate is situated. It is pointed out the need for discussion about the evaluation of the teacher/supervisor activities in clinical psychology. Keywords: Supervision; Clinical psychology; Supervision evaluation scale.
\end{abstract}

\section{Introdução}

A supervisão de estágios é atividade fundamental para a formação de psicólogos. Os estágios supervisionados representam para os estudantes a oportunidade de inserção e transição em novos ambientes e estados de identidade momentos de articulação e integração teóricoprática, experiência indispensável para desenvolvimento e consolidação de diversas competências esperadas de um formando em Psicologia. Os supervisores de estágio são membros do corpo docente dos cursos de Psicologia e deles se espera conhecimento integrado teórico e prático. Entretanto, uma vez credenciado como supervisor, salvo em casos excepcionais, não há revisão desse credenciamento ao longo do tempo, e o supervisor/professor

\footnotetext{
${ }^{1}$ Endereço para correspondência:

E-mail: nancy@unisantos.br
}

realiza seu trabalho praticamente sem uma avaliação externa além daquela de seus alunos/supervisionandos, que, quando ocorre, é de forma assistemática. As relações entre alunos/estagiários e professores/supervisores são diferentes daquelas de alunos e professores - as práticas e os processos de ensino-aprendizagem no âmbito da supervisão são estruturados para além da dinâmica de relações professor/aluno, tomando formas de outras díades como tutor/aprendiz, mestre/discípulo - que são relações mais próximas, marcadas pelo acompanhamento e por avaliações multifacetadas do desenvolvimento das competências do supervisionando.

$\mathrm{Na}$ supervisão, os processos de ensinoaprendizagem têm especificidades referentes aos diferentes atores, cenários e protagonismos envolvidos. No caso da supervisão de Psicologia Clínica, em processos de ensino-aprendizagem clínicos, se inter-relacionam professores/supervisores (com diferentes 
abordagens teórico-metodológicas), estudantesestagiários (que, por vezes, escolhem e, por vezes, são escolhidos pelos supervisores, em processos de seleção), diferentes níveis institucionais (estrutura e funcionamento de cursos de psicologia e de clínicas-escola ou de serviçosescola de psicologia) e clientela atendida (com encaminhamentos, demandas, expectativas e necessidades diversificadas). Refletir sobre um recorte dessa temática da supervisão de estágios, no escopo de atuais discussões sobre formação em Psicologia, advindas da recente implantação das Novas Diretrizes Curriculares para os cursos de psicologia no Brasil (Conselho Nacional de Educação CNE, 2004) é a proposta deste artigo. Após breves e introdutórias considerações sobre o conceito de supervisão no âmbito de supervisão de estágio, será apresentado um estudo sobre percepção de estagiários de Psicologia Clínica acerca de seu supervisor e da experiência da supervisão.

São diversificadas e complexas as funções dos supervisores, no processo de ensino-aprendizagem em psicologia clínica: funções para desenvolvimento de atitudes clínicas em alunos iniciantes numa tarefa que envolve fatores teóricos, técnicos e pessoais. Numa vertente psicanalítica, diz Szecsödy (1990) que as funções do supervisor seriam relativas: 1) ao incentivo da aliança de aprendizagem, para apoiar o desejo de aprender do supervisionando; 2) à manutenção de um setting de trabalho; 3) à compreensão do supervisionando e fazer-se compreender por ele; 4) à identificação do principal conflito do material trazido pelo supervisionando e correspondente formulação de hipóteses compreensíveis; 5) ao auxílio ao supervisionando para reconhecer a resistência e transferência na interação com o paciente, bem como reconhecer suas manifestações contratransferenciais; e 6) ao reconhecimento de suas próprias reações contratransferenciais em relação ao supervisionando.

Fleming e Benedek (1966) afirmam ser fundamental que se desenvolva uma "aliança de aprendizado" entre supervisor e supervisionando, para a eficiência do processo de supervisão. Para tanto, se faz necessário que o supervisor tenha em mente múltiplas funções que pode vir a exercer durante $O$ relacionamento com seu supervisionando (Vollmer \& Bernardi, 1996). Tais funções incluem desde a gerência do processo de supervisão, a atuações como modelo de identificação, ensino de procedimentos técnicos, transmissão de conhecimentos teóricos, e até a representação da instituição a qual pertence.

Binder \& Strupp (1997) historiam, em relação à psicanálise, que Freud se reunia regularmente com seus discípulos iniciais para ensino, supervisão e análise, num modelo no qual os papéis de professor e analista e de aluno e paciente se confundiam; em 1920 com o estabelecimento de institutos de psicanálise e a fundação das sociedades psicanalíticas, a supervisão se formalizou e passou a ser exigida como parte do que é denominado o tripé psicanalítico: estudos teóricos, supervisão e tratamento pessoal (Albuquerque, 1995; Fuks, 2002). A supervisão assume um lugar de continência ao psicoterapeuta iniciante, possibilitando reflexões críticas sobre sua atitude profissional, sua ética e sua eficiência (Peres, 1998), sendo um espaço de discussão e criação de novas possibilidades de pensar (Berti, 2002; Marques, 2000); é um lócus de ensinoaprendizagem que, envolvendo supervisor e supervisionado, desperta sentimentos em ambos (Zaslavsky, Nunes \& Eizirik, 2003), mediante processos de comunicação tanto conscientes como inconscientes.

No contexto da formação do psicólogo, a supervisão ocorre em caráter obrigatório nas clínicas-escola ou em serviços de psicologia integrados a cursos de graduação, tendo em vista a Lei 4.119, artigo 16 (27/08/1962) (Brasil, 1962), que dispõe sobre os cursos de formação em psicologia e regulamenta a profissão do psicólogo, exigindo para funcionamento dos cursos de psicologia a existência de serviços clínicos, entre outros.

Em recente revisão da literatura brasileira sobre supervisão em psicanálise e psicoterapia psicanalítica, a partir de análise de periódicos científicos referenciados entre 2001 e 2006, Saraiva e Nunes (2007) concluíram que há poucos estudos sobre o tema com formato de pesquisa empírica; na citada revisão, as autoras mostraram que os artigos analisados apresentavam poucas discussões sobre assuntos como: passos do supervisor e do supervisionando para atingir os objetivos desejados, mudanças nas técnicas e estratégias da supervisão para patologias na atualidade, controle da supervisão, e dificuldades 
relativas a consentimento informado em artigos com exemplos de caso.

Ainda que vista como indispensável, pode-se afirmar que a temática da supervisão é pouco discutida (Albuquerque, 1995; Fuks, 2002), sendo a maior parte da literatura pertinente, segundo Binder e Strupp (1997), teórica e clínica, embora se possam documentar publicações com pesquisa empírica a esse respeito (Berti, 2002). A supervisão sob a ótica do supervisionando é a porção ainda menos investigada no tema (Rocha, 2001).

\section{Supervisão em clínica-escola: estudo sobre a ótica de} estagiários de psicologia clínica

As autoras pertencem a um grupo de trabalho da Associação Nacional de Pesquisa e PósGraduação em Psicologia - ANPEPP que se dedica à pesquisa sobre clínicas-escola de psicologia. Estudos sobre supervisão de estágios compõem um amplo quadro de trabalhos de investigação que vêm sendo realizados pelo grupo.

O estudo apresentado e discutido no presente artigo privilegia o vértice do aluno/estagiário de curso de graduação de psicologia, dentre diferentes dimensões da investigação sobre supervisão de estágio, como a das partes diretamente envolvidas (o supervisor/professor, o aluno/estagiário, a clientela) e a dos objetivos de trabalho (a formação do aluno, o atendimento oferecido). O estudo teve com objetivo principal a identificação de elementos manifestos da imagem acerca do supervisor e da experiência da supervisão de estágio em Psicologia Clínica. Para tanto, por meio da Escala sobre Experiência de Supervisão na Ótica de Estagiários de Psicologia Clínica, foram exploradas percepções sobre a supervisão em estudantes de nono semestre de curso de psicologia de uma Instituição de Ensino Superior (IES), de turmas de ingressos em 2002.

\section{Sobre o delineamento, instrumento, sujeitos e procedimentos do estudo}

Após apresentação da proposta a instâncias de gestão acadêmica (coordenações do curso e da clínica-escola, e supervisores de estágio) e aos estudantes-estagiários, e seguidos os devidos procedimentos de cuidado de ética de pesquisa com seres humanos (com aplicação de TCLE), que prestava informações sobre o estudo e investigadores, garantia anonimato sobre a identidade dos respondentes, além do direito de desistência da participação na pesquisa a qualquer momento, foram selecionados (por interesse, disponibilidade e aceite) e pesquisados 56 alunos matriculados em $9^{\circ}$ semestre de curso de graduação em psicologia, de Instituição de Ensino Superior da cidade de Santos (SP).

A Escala sobre Experiência de Supervisão na Ótica de Estagiários de Psicologia Clínica, de tipo Likert de cinco pontos, composta por 27 itens (Anexo 1) - instrumento adaptado de um questionário canadense (Nigan, Cameron \& Leverette, 1997) utilizado por Rocha (2001) - foi aplicada aos participantes mediante autoadministração coletiva. A escala encontra-se em processo de validação brasileira realizada por grupo de trabalho da ANPEPP.

\section{Sobre os participantes}

Quando pesquisados, em final de junho de 2006, os alunos, com predomínio do sexo feminino, estavam no início de formação em atendimentos clínicos, sob supervisão em diferentes abordagens teórico-metodológicas, há pelo menos um semestre letivo. Realizavam estágio obrigatório de psicologia clínica, em psicoterapia ou psicodiagnóstico, com atendimentos semanais de crianças, adolescentes, adultos e famílias, feitos na clínica-escola do curso. A partir dos critérios de seleção por interesse no supervisor (e modelo de supervisão) e classificação por histórico escolar (notas), os alunos estavam distribuídos em grupos para nove supervisores dos campos/linhas: psicodignóstico (1), psicodrama (1), psicoterapia analítica (1), psicoterapia cognitivocomportamental (1), psicoterapia fenomenológicoexistencial (2), e psicoterapia psicanalítica (3). As supervisões semanais eram feitas em grupos de cinco a oito alunos, e cada aluno/estagiário recebia supervisão de meia hora-aula para a discussão/orientação/supervisão do(s) atendimento(s) que havia realizado na semana anterior.

\section{Resultados e discussões}

Considerando o número pequeno de sujeitos investigados, alguns resultados estão apresentados de forma descritiva, por freqüências (em porcentagens) de alguns itens da escala. Esses dados estarão agrupados em temas referentes à 
imagem do supervisor (itens relativos a nível afetivo-relacional) e a fatores sugestivos de possível influência da linha teórica na composição da percepção do estagiário.

\section{Sobre a imagem do supervisor (nível afetivo- relacional)}

Sete dentre os itens da escala referem-se à imagem que o estagiário tem do supervisor, em seus aspectos afetivo-relacionais (itens 1, 4, 5, 7, 23, 24, 27). São eles:

1. Seu supervisor apóia seu desejo de aprender?

4.Você se sente compreendido pelo seu supervisor?

5. O seu supervisor aceita os erros que você faz?

7.Você se sente compreendido pelo seu supervisor?
23. Com que freqüência você teve a experiência com um supervisor que tinha uma idéia prestabelecida de que as coisas devem ser feitas apenas da sua maneira?

24. Alguma vez você se achou na posição de ter de "fincar o pé" contra seu supervisor?

27. Você sente que seu supervisor tem confiança nas suas habilidades como psicoterapeuta?

Segundo a freqüência da pontuação das respostas a esses itens da escala e a semântica da avaliação embutida nesses itens, as respostas foram agrupadas em avaliação: negativa, mediana e positiva do supervisor, em seus aspectos afetivorelacionais.

A Figura 1 expõe as freqüências das respostas a esses itens.

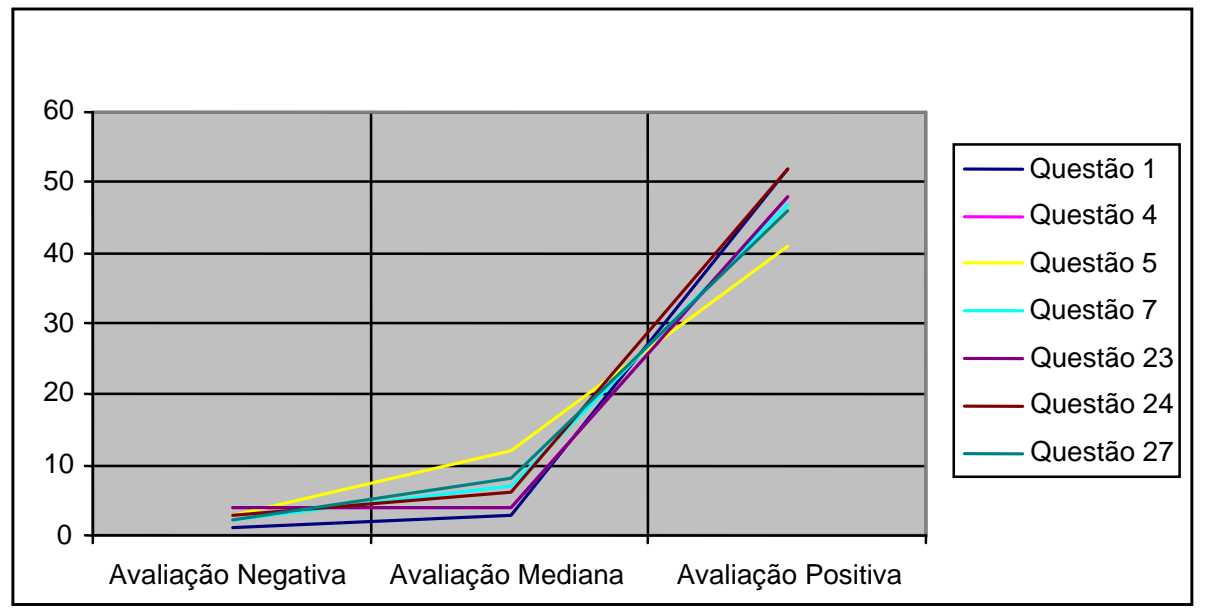

Figura 1 - Quanto à imagem do supervisor

Como pode ser observado, houve grande positividade quanto à imagem do supervisor, aspecto que será a seguir discutido quanto: a características do processo ensino-aprendizagem clínicas (em especial quanto à forma de avaliação utilizada), a possíveis idealizações e ao momento de transição aluno-profissional em que o supervisionando se encontra, que chamaremos de um momento de transição ecológica.

\section{O supervisor como um professor/bom avaliador}

O tipo de processo ensino-aprendizagem clínico que ocorre na supervisão (contexto de ensino-aprendizagem próprio da prática profissional supervisionada) tem características muito particulares.
O supervisor avalia o progresso do aprendizado clínico do estagiário com a responsabilidade pelo cuidado adequado ao paciente atendido (Jacobs, David e Meyer, 1995). A supervisão é uma relação avaliativa: em relação ao supervisionando e à qualidade dos serviços oferecidos aos clientes, que necessita de tempo para possibilitar crescimento e aprendizado do psicoterapeuta em formação (Bernard e Goodyear, 1992; Silva, 2003). No caso de estágios supervisionados específicos nos cursos de graduação de psicologia, esse tempo dura, em geral, dois semestres letivos.

Além de monitorar e supervisionar o serviço que está sendo oferecido à clientela, ao professor/supervisor cabe acompanhar e avaliar o 
desenvolvimento do aluno/estagiário, em domínios que avançam para além dos campos que podemos chamar cognitivos, da ordem do teórico, para a integração com os campos das habilidades técnicas e de atitude clínica. $O$ processo de ensino-aprendizagem clínico teria claramente uma natureza cognitivo-afetiva (Vollmer e Bernard, 1996; Szecsödy, 1997).

Com respeito à atitude clínica a ser desenvolvida nos estágios de psicologia clínica, Aguirre e cols. (2000) consideram-na como uma experiência subjetiva que fica objetivada na relação com o cliente. Para essas autoras, a atitude clínica é representante de um fenômeno interno complexo em que concorrem: conhecimento teórico, experiências pessoais, identificações, fantasias sobre o papel do psicólogo, possibilidades de experimentação e investigação de vivências interiores, e capacidade de conter ansiedades e de preservar limites da própria identidade no contato com o outro. A avaliação desse complexo fenômeno, no qual muitas vezes conteúdos emocionais convertem-se em instrumentos de trabalho, necessariamente também envolve certa complexidade.

Abuchaim (1996) comenta sobre a existência de uma polarização na bibliografia específica de orientação analítica, entre duas atitudes na supervisão: uma chamada de didática e outra de experiencial. $\mathrm{Na}$ primeira, o supervisor age estritamente como professor, para explicar, corrigir e sugerir, tornando-se um modelo para uma identificação por parte do supervisionando, sem lidar com situações propriamente afetivas do supervisionando, o que seria realizado em um tratamento/psicoterapia pessoal. $\mathrm{Na}$ atitude experencial, a função do supervisor inclui facilitar o crescimento pessoal do supervisionando, de forma que problemas em aprender e compreender suas próprias reações com os pacientes e com o supervisor são tratados com o próprio supervisor. Dessa maneira, as reações contratransferenciais (estados emocionais inconscientes, expressos na conduta do supervisionando) com os pacientes e com o supervisor seriam elaboradas na própria supervisão.

A avaliação do desenvolvimento do supervisionando, com tratamento mais ou menos direto pelo supervisor, procura quase sempre considerar a conquista de independência, um processo de aprendizagem para além do processo de supervisão (Schlesinger, 1981). O supervisionando deverá ter desenvolvido habilidades e competências clínicas em sua formação, e conquistado necessárias uniões de teoria e de prática, interdependentes que na supervisão têm seu ponto crucial de aprendizado (Haley, 1998).

Os estágios de psicologia clínica em cursos de graduação em psicologia do Brasil acontecem como unidades curriculares que exigem avaliações com exigências formais para legitimidade da formação do graduando. Para tanto, diferente de unidades curriculares como disciplinas ou módulos, são bastante freqüentes as avaliações por conceitos, sem uso de prova-nota: um tipo de avaliação identificada com orientação, retroalimentação, suporte técnico e teórico, e muitas vezes até pessoal. Esse tipo de avaliação, que de toda forma é mais pessoal, pode compor uma imagem do supervisor mais positiva, como encontrada nos dados do estudo de referência.

Mesmo que utilizando estilos e técnicas diversos, supervisores de psicologia clínica, geralmente solicitam de seus alunos/supervisionandos relatórios e/ou narrativas semanais sobre o atendimento realizado. Esse acompanhamento feito em pequenos grupos de alunos (freqüentemente muito menores do que os grupos de uma sala de aula), pode favorecer e promover um pensar sobre as experiências, numa vinculação direta com a prática, sem necessidade da prova-verificação (com conceito/nota), pouco usada (ou efetiva) para avaliar habilidades e atitudes clínicas.

Essa avaliação formativa, processual e dinâmica, que se distingue de tradicionais avaliações teóricas por prova-nota, pode levar os alunos/supervisionandos a identificarem o supervisor com uma imagem positiva de um professor/bom-avaliador. Ao mesmo tempo, tal condição de avaliação pode favorecer o surgimento e a manutenção de um tipo de relação mais dependente para negação e dissimulação de eventuais problemas na relação com o professorsupervisor. Nesse contexto de avaliação formativa e mais subjetiva, o aluno-supervisionando não dispõe do recurso de uma avaliação objetiva (prova-nota) e seus dispositivos (revisão de prova com outro professor, por exemplo) para possíveis discussões e/ou contraposições com o supervisor.

É claro que a relação da supervisão é uma 
relação assimétrica, na qual o supervisor encontrase numa posição superior, com o pressuposto que possua, em termos clínicos, um mundo interno mais elaborado e com condição básica de saúde mental (Brito, 1999). No entanto, o supervisor de estágios de psicologia clínica tem sua face pedagógica (ensino e avaliação) inserida numa estrutura de graduação que possui parâmetros curriculares, como cronogramas letivos, que têm natureza um pouco diferente das exigências e condições para formação de psicoterapeutas ou analistas em instituições psicanalíticas, por exemplo.

Nesse sentido, a alta positividade denotada no estudo a que nos reportamos, para além da percepção de qualidades e/ou virtudes do supervisor, pode embutir negação de conflitos que, se percebidos e/ou manifestados, colocariam $\mathrm{o}$ aluno/supervisionando em situação mais vulnerável diante do supervisor do que de um professor.

\section{O supervisor como terapenta idealizado}

Cabe principalmente aos supervisores clínicos, em suas diferentes abordagens teóricotécnicas, auxiliar os supervisionandos no desenvolvimento de experiências subjetivas necessárias para o estabelecimento da identidade de psicólogo - auxílio dentro de parâmetros de supervisão, que por vezes pode estar pouco distinguido de parâmetros de psicoterapia, ou até em tarefas nas quais papéis de terapeuta e professor se misturam (Brito, 1999).

A alta positividade da imagem do supervisor verificada nos casos estudados, assim, pode ser devida a mecanismos de idealização e de identificação com o professor/supervisor na figura de um psicólogo ou psicoterapeuta idealizado. Nesse sentido, o campo de atuação dos supervisores poderia ser entendido e confundido com o campo do psicoterapeuta, sendo os professores/supervisores tratados mais como psicólogos do que como professores, e mais fortemente passíveis de mecanismos de idealização e identificação.

Num contexto psicanalítico, Pechanski (1996) afirma que o supervisor oferece-se como objeto de identificação ao participar da formação do candidato à analista, corrigindo distorções, apontando acertos e erros, trocando experiências, e sendo continente diante da ignorância e inexperiência do candidato.

Dessa forma, o supervisor de estágios clínicos pode ser confundido com um terapeuta, e terapeuta idealizado considerando a insegurança e inexperiência do supervisionando para realizar tarefas de psicólogo, com apenas um semestre letivo (cerca de quatro meses) de supervisão.

\section{O supervisor como figura necessária para a transição ecológica aluno-profissional}

A alta positividade da imagem do supervisor também pode estar associada ao papel do professor/supervisor, mais claramente identificado como o "profissional de psicologia", importante figura de apoio para as vicissitudes da mudança de papéis e também de ambientes, como uma "transição ecológica" (Bronfenbrenner, 1996) experimentada pelos alunos em sua passagem da condição de alunos para a de profissionais.

Os estagiários desempenham papéis e funções de psicólogos, embora ainda não o sejam, mas estejam transitando para sê-lo. Estados de insegurança são constantes nessa transição, tanto diante dos clientes atendidos e dos ambientes institucionais de atendimento, como diante do supervisor, dos colegas de grupo de supervisão, e especialmente diante de si mesmos (Aguirre \& cols., 2000).

Para contenção desses estados, propiciados pela inexperiência e por fantasias acerca do papel de psicólogo, figuras dos professores/supervisores podem funcionar como esperados continentes de apoio. Nesse sentido, supervisores podem ser avaliados muito positivamente, já que amparam estados de insegurança dos alunos/estagiários, próprios da transição de papéis do início da formação da identidade profissional.

\section{Sobre os fatores sugestivos de influência da linha teórica}

Quatro dentre os itens da escala são sugestivos de influência da linha teórico-técnica do supervisor compondo a ótica dos supervisionandos acerca de seus supervisores de psicologia clínica (itens 12, 13, 17 e 21). São eles:

12. O seu supervisor lhe dá exemplos de seus próprios pacientes para ajudá-lo a lidar com os dilemas dos seus pacientes?

13. O seu supervisor encoraja a exploração de aspectos contratransferenciais (aspectos pessoais mobilizados pela interação com o paciente que 
possam interferir no processo psicoterapêutico)?

17. Com que freqüência pediram para que você preparasse supervisão verbalmente ou por escrito? 21. O seu supervisor lhe ajuda com seus dilemas dizendo algo do tipo "o que eu faria nesta situação é isto..."?

A Figura 2 apresenta dados de freqüência de respostas a esses itens.

\section{Frequentemente}

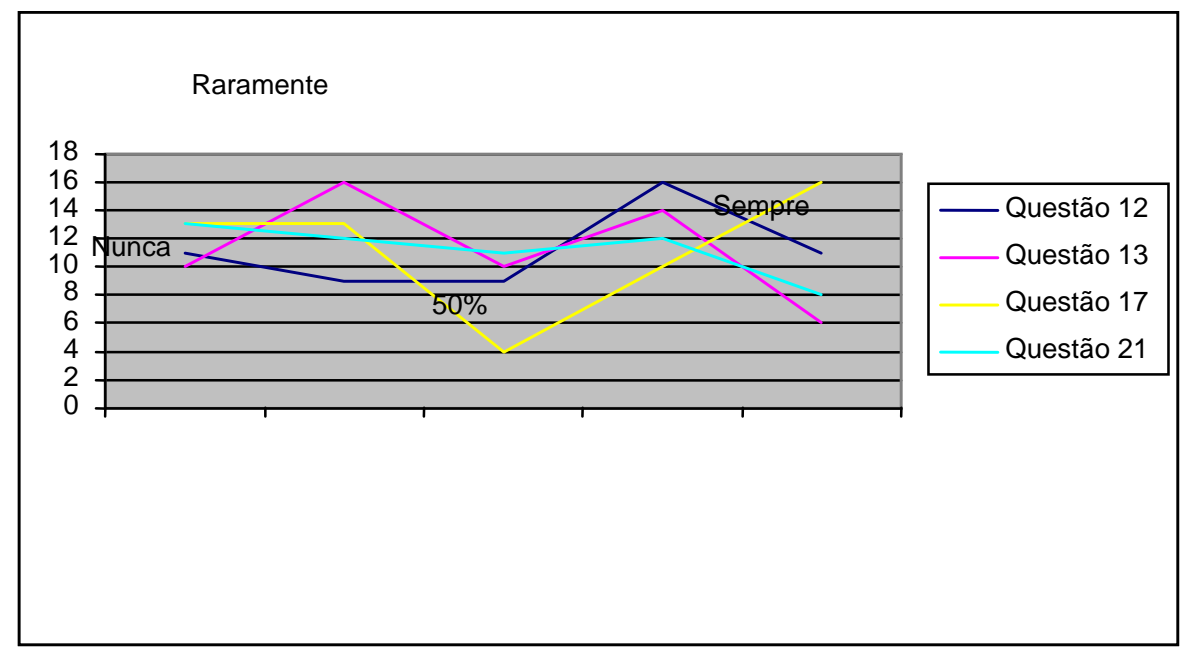

Figura 2 - Quanto à possível influência da linha teórico/técnica

Pelo observado na Figura 2, as freqüências de respostas tiveram tendências semelhantes nesses quatro itens. $O$ item 13 continha a expressão "aspectos contratransferenciais", e o uso da terminologia específica (contratransferência) pode ter vinculado a questão ao sentido de que o supervisor seria de uma linha psicanalítica, com possível influência nos resultados.

linhas

Esses dados sugerem que diferenças nas professor/supervisor (sugeridas pelos quatro itens da escala) pouco influenciaram a imagem que possuem seus alunos/supervisionandos. Esse dado parece reafirmar que a alta positividade da avaliação independe da abordagem teórico-técnica e pode ser decorrente dos fatores comentados na sessão anterior.

\section{Considerações finais}

O limite do estudo apresentado é pequeno, visto o número de investigados e a natureza do instrumento utilizado. Nessa dimensão avaliada, os alunos/supervisionandos pesquisados indicaram possuir uma visão muito positiva - com sugestão de idealizada ou até pouco crítica - de seus professores/supervisores de Psicologia Clínica. Sem pretensão de maior generalização visto o limite referido, esses resultados podem indicar que mecanismos de idealização do professorsupervisor de psicologia clínica devam ser considerados em experiências de estágios acadêmicos de cursos de graduação em Psicologia, não apenas como ocorrências particulares de supervisionandos, mas como fenômenos constituintes da própria experiência dessa categoria de supervisão.

O supervisor de psicologia clínica pode ser um professor idealizado, menos avaliado realisticamente em suas qualidades pedagógicas e mais poupado de críticas negativas, por ser uma figura acadêmica facilmente identificada por seus alunos/supervisionandos como um profissional psicólogo. A qualidade da interação com esse psicólogo/professor vai facilitar os processos de assimilação de atributos profissionais cognitivos, afetivos, técnicos e éticos - aqueles necessários para conquista da diferenciação de papéis de estudante e de estagiário/futuro profissional. $\mathrm{O}$ supervisor é professor próximo e disponível no apoio para a transição do mundo da universidade 
para o mundo do trabalho.

Dado esse fundamental papel educativo do supervisor de estágio em psicologia clínica e uma possível idealização que o cerca, consideramos a necessidade de ampliação e aprofundamento das discussões sobre a complexidade da experiência acadêmica da supervisão clínica para a formação do psicólogo.

\section{Referências}

Abuchaim, C. M. (1996). A relação ensino aprendizagem em supervisão de psicoterapia de orientação analitica. VII Jornada Paulo Guedes, Fundação Universitária Mário Martins. Porto Alegre.

Aguirre, A. M. de B., Herzberg, E., Pinto, E. B., Becker. E., Carmo, H. M. S. e Santiago, M. D. E. (2000). A formação da atitude clínica no estagiário de psicologia. Psicol. USP, 11, (1).

Albuquerque, J. D. C. (1995). Sobre a supervisão algumas considerações. Cad. Temp. Psic., Rio de Janeiro, 1, 99-104.

Bernard \& Goodyear (1992). Fundamentals of clinical supervision. Boston: Allyn \& Bacon.

Berti, T. R. (2002). A importância da supervisão na formação da identidade do psicoterapeuta. Revista Brasileira de Psicoterapia, 4, 139-153.

Binder, J. \& Strupp, H. H. (1997). Handbook of psychotherapy supervision. USA, John Wiley \& Sons Inc.

Brasil (1962). Lei $\mathrm{n}^{\circ}$ 4.119, que dispõe sobre a formação em Psicologia e regulamenta a profissão de Psicólogo. Capítulo IV, Artigo 16, p. 3 . Disponível em <http://www.pol.org.br/legislacao/pdf/lei_n_ 4.119.pdf $>$ Acesso em 6 de junho de 2005.

Brito, C. L. S. (1999). A transmissão do conbecimento psicanalitico através da supervisão. Dissertação de mestrado. Porto Alegre: Pontifica Universidade Católica do Rio Grande do Sul.

Bronfenbrenner, U. (1979/1996). Ecologia do desenvolvimento bumano: experimentos naturais $e$ planejados. Porto Alegre: Artmed.

CNE (2004). Conselho Nacional de Educação, Câmara de Educação Superior. Diretrizes curriculares nacionais para os cursos de graduação em Psicologia, Resolução n ${ }^{\circ} 8$ de 7 de maio de 2004.

Fleming, J. \& Benedek, T. (1996). The Psychoanalytic Supervision. Nova Iorque: Grune \& Stratton.

Fuks, L. B. (2002). Formação e supervisão. Psicanálise e Universidade, 16, 79-91.

Haley, J. (1998). Aprendendo e ensinando terapia. Porto Alegre: Artmed.

Jacobs, D., David, P. \& Meyer, D. J. (1995) The supervisory encounter: a guide for teachers of psychodynamic psychotherapy and psychoanalysis. Nova Iorque: Yale University.

Marques, S. F. (2000). Narrativa e supervisão na psicanálise. Psychê, 4 (5).

Nigan, T., Cameron, P. M. \& Leverette, J. S. (1997) Impasses in the supervisory process: A resident's perspective. American Journal of Psychoterapy, 51, 252-272.

Pechansky, I. (1996). Vicissitudes da supervisão psicanalítica. Revista de Psicanálise, 3, (2), 295302.

Peres, W. S. (1998). A instituição do estágio: uma reflexão sobre os analisadores. Psicologia em Estudo, 3 (2), 163-176.

Rocha, N. S. (2001). Supervisão em psicoterapia de orientação analítica: a perspectiva de uma amostra de supervisionados de Porto Alegre, Brasil. Revista Brasileira de Psicoterapia, 3 (3), 213-228.

Saraiva, L. A. \& Nunes, M. L. T. (2007). A supervisão na formação do analista e do psicoterapeuta psicanalítico. Estudos de Psicologia, 12, 259-268.

Silva, J. F. da G. (2003). Comunicação e aprendizagem na supervisão analítica. Cadernos de Psicanálise - SPCRJ, 19 (22), 113-133.

Schlesinger, H. J. (1991). General Principles of Psychoanalitic Supervision. In Wallerstein, R.S. (Org.). Becoming a Psychoanalyst. Nova Iorque: International University Press.

Szecsödy I. (1997). (How) Is learning possible in supervision? Em Martindale B. Supervision and its vicissiyudes. London: Karnak Books.

Szecsödy, I. (1990). The Significance and 
Importance of Supervision in Psychotherapy Training. Psychotherapy and Psychosomatics, 53, 86-92,

Vollmer Filho, G. \& Bernardi, R. (1996). As funções múltiplas do supervisor, os seus relacionamentos com o supervisionando, o analista do supervisionando, o paciente, o quadro de referência teórico e a instituição de treinamento. Revista de Psicanálise da Sociedade Psicanalitica de Porto Alegre, 3, 283-293.
Zaslavsky, J., Nunes, M. L. T. \& Eizirik, C. L. (2003). A supervisão psicanalítica: revisão e uma proposta de sistematização. Revista de Psiquiatria do RS, 25 (2), 297-309.

Recebido em novembro de 2007 Reformulado em maio de 2008 Aprovado em setembro de 2008

Sobre as autoras:

Nancy Ramacciotti de Oliveira-Monteiro é psicóloga, possui mestrado pela Pontifícia Universidade de São Paulo (Psicologia Social) e doutorado pela Universidade de São Paulo (Psicologia Social). Atualmente é professora adjunta da Universidade Federal de São Paulo (UNIFESP) e chefe do departamento Saúde, Educação e Sociedade, da UNIFESP-BS. Tem experiência em Psicoterapia, Supervisão de psicologia clínica (psicodiagnóstico) e pesquisa sobre desenvolvimento humano (maternidade de adolescentes).

Maria Lúcia Tiellet Nunes é psicóloga, possui mestrado pela Universidade Federal da Paraíba (Psicologia Social) e doutorado pela Freie Universitat Berlin (Psicologia Tratamento e Prevenção). Atualmente é professora titular e coordenadora do Programa de Pós-Graduação em Psicologia da Pontifícia Universidade Católica do Rio Grande do Sul. Tem experiência em pesquisa e em psicoterapia, atuando principalmente nos temas: psicoterapia, crianças, psicanálise, materiais terapêuticos, psicopatologia e relação mãe-bebê. 
Anexo 1 - Escala sobre experiência de supervisão na ótica de estagiários de Psicologia Clínica (Rocha, 2001)

$$
(\mathrm{N} / \mathrm{R} / 50 \% / \mathrm{F} / \mathrm{S})
$$

1. Seu supervisor apóia seu desejo de aprender?

2. Seu supervisor ajusta seus conhecimentos para seu nível de treinamento e experiência?

3. Você descreveria seu supervisor como um bom modelo?

4. Você se sente respeitado pelo seu supervisor?

5. O seu supervisor aceita os erros que você faz?

6. O quanto você se sente criticado pelo seu supervisor?

7. Você se sente compreendido pelo seu supervisor?

8. Com que freqüência seu supervisor impõe a você sua própria "prescrição psicoterapêutica"?

9. Seu(s) supervisor(es) está(ão) disponíveis quando ocorre alguma crise com seu paciente?

10. Você se sente obrigado a concordar com as idéias e pensamentos de seu supervisor por ter medo de ser desvalorizado?

11. Alguma vez você ficou chateado com seu supervisor porque ele não lhe apoiou emocionalmente?

12. O seu supervisor lhe dá exemplos de seus próprios pacientes para ajudá-lo a lidar com os dilemas dos seus pacientes?

13. O seu supervisor encoraja a exploração de aspectos contratransferenciais (aspectos pessoais mobilizados pela interação com o paciente que possam interferir no processo psicoterapêutico)?

14. Com que freqüência você lembra de uma situação em que o tratamento de um paciente não progrediu por mais de 3-4 semanas?

15. Com que freqüência você lembra que a supervisão de um paciente não trouxe nenhuma informação nova por mais de 3 ou 4 semanas?

16. Com que freqüência você sente que aprendeu algo na supervisão?

17. Com que freqüência pediram para que você preparasse supervisão verbalmente ou por escrito?

18. Quão livre você é para expressar uma opinião diferente da de seu supervisor?

19. Com que freqüência você sentiu o supervisor invadindo sua vida pessoal?

20. Alguma vez você se sentiu envergonhado ou humilhado pelos comentários do seu supervisor?

21. O seu supervisor lhe ajuda com seus dilemas dizendo algo do tipo "o que eu faria nesta situação é isto..."?

22. Com que freqüência você sentiu que seu supervisor era "só trabalho" e deixou pouco espaço para trocas mais informais?

23. Com que freqüência você teve a experiência com um supervisor que tinha uma idéia prestabelecida de que as coisas devem ser feitas apenas da sua maneira?

24. Alguma vez você se achou na posição de ter de "fincar o pé" contra seu supervisor?

25. Alguma vez você se percebeu não dizendo o que realmente acontecia na terapia (atendimento) do seu paciente?

26. Você acha que seu supervisor é fundamentalmente um crítico de seu trabalho?

27. Você sente que seu supervisor tem confiança nas suas habilidades como psicoterapeuta? 